\title{
Adaptive Finite-Time Congestion Control for Uncertain TCP/AQM Network with Unknown Hysteresis
}

\author{
Weimin Zheng, Yanxin Li $(\mathbb{D}$, Xiaowen Jing, and Shangkun Liu \\ College of Computer Science and Engineering, Shandong University of Science and Technology, Qingdao 266590, China \\ Correspondence should be addressed to Yanxin Li; lyx94lyx@126.com
}

Received 8 May 2020; Revised 24 June 2020; Accepted 6 July 2020; Published 27 July 2020

Guest Editor: Lei Xie

Copyright (C) 2020 Weimin Zheng et al. This is an open access article distributed under the Creative Commons Attribution License, which permits unrestricted use, distribution, and reproduction in any medium, provided the original work is properly cited.

\begin{abstract}
The issue of adaptive practical finite-time (FT) congestion control for the transmission control protocol/active queue management (TCP/AQM) network with unknown hysteresis and external disturbance is considered in this paper. A finite-time congestion controller is designed by the backstepping technique and the adaptive neural control method. This controller guarantees that the queue length tracks the desired queue in finite-time, and it is semiglobally practical finite-time stable (SGPFS) for all the signals of the closed-loop system. At last, the simulation results show that the control strategy is effective.
\end{abstract}

\section{Introduction}

Recently, the communication network based on TCP has been rapidly developed and widely used. However, the congestion of network traffic has become a critical problem in network control. TCP congestion control can not only ward off network collapse and avert locking behavior, but also effectively reduce the probability of control loop synchronization [1]. It is of great significance to maintain the stability and robustness of the TCP network. Since Jacobson proposed the end-to-end TCP congestion control algorithm in 1988 [2], many scholars have conducted more in-depth and detailed research, such as Vegas [3], Sack [4], and New Reno [5], and they all concentrate on end-to-end congestion control based on the end system. However, with the increasing demand of application and the improvement of service quality, the TCP congestion control mechanism of end-to-end is no longer suitable. After that, some scholars have proposed a solution called AQM [6], which is one of the most widely studied congestion solutions at present. It can drop or mark some packets before the router generates a full queue state, so that the TCP source can timely sense the network congestion state and take corresponding measures. The first algorithm is named as random early detection (RED) [7], but RED and its improved algorithms [8, 9] are too complicated for parameter configuration. Based on fluid flow theory, Misra et al. proposed a nonlinear model of TCP/AQM in 2000 [10]. Based on the above model, many researchers have combined control theory to design several congestion schemes, such as P and PI [11], PD [12], and PID [13]. An AQM algorithm is presented on the strength of the fuzzy sliding-mode control method in the nonlinear control method in [14], which improves the control effect of the system. In [15], considering the limited input of the TCP network system, an AQM algorithm is presented on the strength of the sliding-mode control method, which makes the system obtain better asymptotic stability. Tan et al. [16] proposed a congestion control scheme composed of source and link algorithms. Zhang et al. [17] analyzed the TCP/RED model from a time-delay control theory standpoint, and the time-delayed control analyzing techniques explored can be extended to other AQM or AQM-based schemes for their stability analysis. The primal-dual algorithm has been analyzed from the multivariable time-delay control theory standpoint in [18]. The stability analysis can be applied to various TCP/AQM systems other than FAST TCP/DropTail and TCP/AVQ. The AQM controller with specified performance is designed by the backstepping method considering the interference of UDP flow [19]. Due to the complex environment and continuous 
application of the network [20-22], congestion algorithms need to further improve network performance and achieve better congestion control effects.

Nowadays, many scholars have noticed the hysteresis phenomenon widely existing in the nonlinear system. In the process of practical application, the tracking performance of the system has been limited by the hysteresis, which even makes the system unstable $[23,24]$. To reduce the impact of nonlinear system control on the unknown hysteresis in the actuator, more and more scholars have studied the design of the controller in [25-28]. Zhou et al. [25] not only first proposed a new Bouc-Wen hysteresis model, but also put forward an adaptive control method to ensure a good tracking performance by constructing an inverse of compensating hysteresis nonlinearity. For the sake of the stability of the controlled system, Su et al. [26] designed an adaptive control scheme based on the backlash-like hysteresis model. Wang et al. [28] discussed the adaptive stabilization of pure-feedback nonlinear systems, and it not only solved the unknown direction hysteresis, but also eliminated the constraint assumption that the nonlinear function needs to satisfy the linear growth condition by using the characteristic of the Nussbaum function and introducing a virtual controller. Up to now, there are no literature studies for the congestion control of TCP/ AQM networks with unknown hysteresis.

On the other hand, some scholars pay more attention to the FT control because the FT stability has more meaning than infinite-time stability in practical application [29-31]. In practice, the control goal is promising to be realized in a limited time, and the control scheme of infinite time cannot achieve such a goal because they will lead to a long time transient response. The FT stability is different from the asymptotic stability of infinite time, its control method will enable the system to achieve the transient performance quickly, and in FT, the system state variables can be converged in equilibrium. Dorato conducted a comprehensive study on the problem of FT stability and elaborated on the differences between FT stability and asymptotic stability [29]. The Lyapunov theory of FT stability was first put forward in $[30,32]$. The authors in $[33,34]$ proposed some FT control schemes for the nonlinear systems via the Lyapunov stability theory. However, in practice, these FT control strategies cannot satisfy the actual control system with unknown nonlinearity. Wang et al. [34] studied the FT tracking problem with unknown functions. A criterion of SGPFS is set up for the first time by the fuzzy logic system (FLS), and a novel adaptive fuzzy control strategy based on it is presented.

To sum up, this manuscript researches FT congestion control for the TCP/AQM network with unknown hysteresis and external disturbance. The main works are summarized as follows:

(1) Inspired from [35], a newfangled model of TCP is established, which considers the effects of hysteresis input and exogenous disturbance. The model in this work is more general and more exact.

(2) An adaptive FT congestion controller is constructed by combining the backstepping technique and the radial basis function neural networks (RBFNNs) in this paper. This controller guarantees that the queue length tracks the desired queue in FT, and all the signals of the closed-loop system are SGPFS.

(3) The classical FT control strategy requires that the nonlinear function of the controlled system must satisfy the linear growth condition or matching condition [30, 36-38]. In this article, as the nonlinear function is unknown, the studied system cannot satisfy the linear growth condition. Therefore, in the new congestion control strategy, continuity is the only requirement for nonlinear functions. Hence, this scheme is more general.

The surplus of the manuscript is summarized as follows. A network model and advance preliminaries are recommended in Section 2. The main result is shown in Section 3. Section 4 gives a simulation example. Finally, Section 5 draws a conclusion.

\section{Model and Preliminaries}

2.1. TCP/AQM System Model. This article, based on [35], considers the following TCP/AQM network, in which the authors takes account of external interference, and in contrast, the time-delay is neglected:

$$
\left\{\begin{array}{l}
\dot{W}(t)=\frac{1}{R(t)}(1-p(t))-\frac{W(t)}{2} \frac{W(t-R(t))}{R(t-R(t))} p(t-R(t)), \\
\dot{q}(t)=\left\{\begin{array}{c}
-C(t)+\frac{N(t)}{R(t)} W(t)+\omega(t), q>0 \\
\max \left\{0,-C(t)+\frac{N(t)}{R(t)} W(t)+\omega(t)\right\}, q=0
\end{array}\right\}, \\
R(t)=\frac{q(t)}{C(t)}+T_{p},
\end{array}\right.
$$

where $W(t) \in\left[0, W_{\max }\right]$ is the TCP window size, $q(t) \in\left(0, q_{\max }\right]$ is the queue length of the router, $R(t)$ is the round-trip time, $C(t), N(t)$, and $T_{P}$ are the available link capacity, the number of TCP sessions, and the propagation delay, respectively, $p \in[0,1]$ is the probability of packet loss, and $\omega(t)$ is the external disturbance, which can be thought of as unresponsive flows.

Assumption 1. In fact, most Internet routing scenarios change over time slowly. Therefore, we suppose that the parameters $N(t)$ and $C(t)$ are fixed values $[13,39] . N(t)$, $C(t)$, and $R(t)$ can be simply rewritten as $N, C$, and $R$.

Assumption 2. It is bounded for the external disturbance $\omega(t)$ and its derivative.

According to [40], the rate model is adopted by 


$$
\dot{r}(t)=\frac{\dot{w}(t)-r(t) \dot{R}}{R},
$$

with $r(t)=W(t) / R$. It is not hard to conclude that, based on (1) and (2),

$$
\left\{\begin{array}{l}
\dot{r}(t)=\frac{1}{R^{2}}-\frac{1}{R^{2}} p(t)-\frac{r^{2}(t)}{2} p(t)+\frac{r(t)}{R}-\frac{N r^{2}(t)}{R C}-\frac{r(t) \omega(t)}{R C}, \\
\dot{q}(t)=-C+N r(t)+\omega(t) .
\end{array}\right.
$$

Set $x_{1}=q(t), x_{2}=r(t)$, and $u(t)=p(t)$, and the above model can be converted to

$$
\left\{\begin{array}{l}
\dot{x}_{1}=N x_{2}+\omega(t)-C, \\
\dot{x}_{2}=f(x)+g(x) u(t)+\psi(x) \omega(t) \\
y=x_{1}
\end{array}\right.
$$

where $x(t)=\left[x_{1}(t), x_{1}(t)\right]^{T}$ and $f(x)$ and $g(x)$ are as follows:

$$
\begin{aligned}
& f(x)=\frac{1}{R^{2}}-\frac{N}{R C} x_{2}+\frac{x_{2}}{R}, \\
& g(x)=-\frac{1}{R^{2}}-\frac{1}{2} x_{2}^{2}, \\
& \psi(x)=-\frac{x_{2}}{R C} .
\end{aligned}
$$

2.2. Hysteresis Nonlinearity. Consider a modified Bouc-Wen hysteresis in the form of

$$
H(u)=\mu_{1} u+\mu_{2} l .
$$

It is assumed that sign $\left(\mu_{1}\right)=\operatorname{sign}\left(\mu_{2}\right)$ and

$$
\dot{i}=\dot{u}-\varpi|\dot{u}||\iota|^{9-1}-\xi \dot{u}|\iota|^{9}=\dot{u} f(\iota, \dot{u}), \iota\left(t_{0}\right)=0,
$$

where $₫>|\xi|, n>1,|\iota| \leq \bar{\imath}=\sqrt[n]{1 /(\emptyset+\xi)}, \emptyset$ is the shape and amplitude of the hysteresis, and $\vartheta$ represents the smoothness from the initial slope to the asymptote's slope. Define $f(l, \dot{u})$ as

$$
f(\iota, \dot{u})=1-\operatorname{sign}(\dot{u}) 9|\iota|^{9-1} \iota-\xi|\iota|^{9} .
$$

Remark 1. The sign of $\mu_{1}$ determines the direction of hysteresis.

Assumption 3. Without losing generality, assume that $\operatorname{sign}\left(\mu_{1}\right)>0$.

Remark 2. In the process of practical application, the tracking performance of the system has been limited by the hysteresis, which even makes the system unstable. For the TCP/AQM system model (4), the authors take account of the hysteresis nonlinearity in the control system to reduce the influence of the hysteresis input on the system and realize the stability of the controlled system.
2.3. RBFNNs. RBFNNs are applied to identify the unknown nonlinear functions. The RBFNNs can be written as

$$
f_{n n}(\varsigma)=\Upsilon^{T} \hbar(\varsigma) \text {, }
$$

where $\quad \varsigma \in \Omega_{\varsigma} \subset \mathscr{R}_{q}$ is the input vector, $\Upsilon=\left(\Upsilon_{1}, \Upsilon_{2}, \ldots, \Upsilon_{\aleph}\right)^{T} \in \mathscr{R}_{\aleph}$ is the weight vector, $\aleph>1$ represents the number of RBFNNs nodes, and $\hbar(\varsigma)=\left[\hbar_{1}(\varsigma), \hbar_{2}(\varsigma), \ldots, \hbar_{\aleph}(\varsigma)\right]^{T} \in \mathscr{R}_{\aleph}$ denotes the basis function vector. Select the Gaussian basis function $\hbar_{i}(\varsigma)$ as follows:

$$
\hbar_{i}(\varsigma)=\exp \left[-\frac{\left(\varsigma-\kappa_{i}\right)^{T}\left(\varsigma-\kappa_{i}\right)}{v^{2}}\right], \quad i=1,2, \ldots, \aleph,
$$

where $v$ is the width of the Gaussian function and $\kappa_{i}=$ $\left[\kappa_{i 1}, \ldots, \kappa_{i q}\right]$ is the center of the receptive field.

Lemma 1 (see [41]). Define a continuous function $f(\varsigma)$ on a compact set $\Omega$. Then, for $\forall \varepsilon>0$, the following holds:

$$
f(\varsigma)=\Upsilon^{* T} \hbar(\varsigma)+\epsilon(\varsigma),
$$

where

$$
\Phi=\arg \min _{\Upsilon}\left[\sup _{\varsigma \in \Omega}\left|f(\bar{x})-\Upsilon^{T} \hbar(\varsigma)\right|\right], \epsilon(\varsigma) \leq \varepsilon .
$$

Definition 1 (see [42]). Consider the following system:

$$
\dot{\wp}=f(\wp) \text {, }
$$

where $\wp$ represents the state vector. The origin of system (13) is SGPFS if all $\wp\left(t_{0}\right)=\wp_{0}$, and there exist $\delta>0$ and a setting time $0<T\left(\wp_{0}, \delta\right)<\infty$ such that $\|\wp(t)\| \leq \delta$ when $t \geq t_{0}+T$.

Assumption 4. There exists an unknown constant $b_{2}>0$ such that

$$
b_{2} \leq|g(x)| \text {. }
$$

Lemma 2 (see [43]). For any real variables $x_{0}$ and $y_{0}$ and constants $\imath>0, \xi>0$, and $\mu>0$, the following inequality is satisfied:

$$
|\phi|^{\xi}|\psi|^{\xi} \leq \frac{1}{1+\xi} \mu|\phi|^{1+\xi}+\frac{\xi}{1+\xi} \mu^{1 / \mu}|\psi|^{1+\xi} .
$$

Lemma 3 (see [41]). For any real number $l_{i}, i=1,2, \ldots, n$, when $\zeta \in(0,1)$ and $\lambda \in(0,2)$, it holds

$$
\begin{aligned}
& \left(\left|l_{1}\right|+\cdots\left|l_{n}\right|\right)^{\zeta} \leq\left|l_{1}\right|^{\zeta}+\cdots+\left|l_{n}\right|^{\zeta}, \\
& \left(\left|l_{1}\right|^{2}+\cdots+\left|l_{n}\right|^{2}\right)^{\lambda} \leq\left(\left|l_{1}\right|^{\lambda}+\cdots+\left|l_{n}\right|^{\lambda}\right)^{2} .
\end{aligned}
$$

Lemma 4 (see [44]). Consider system (13), and suppose that there is a $C^{1}$ function $V(\wp)$ and $V(\wp)$ with $V(0)=0$ is positive definite on $D$, one has

$$
\dot{V}(\wp) \leq-\tau V^{\zeta}(\wp)+\varrho,
$$


where $\tau>0,0<\zeta<1$, and $0<\varrho<\infty$. Then, the trajectory of system $\dot{\wp}=f(\wp)$ is SGPFS.

Proof. According to equation (17), for $0<\varnothing<1$, the following inequality holds:

$$
\dot{V}(\wp) \leq-\varnothing \tau V^{\zeta}(\wp)-(1-\varnothing) \tau V^{\zeta}(\wp)+\varrho .
$$

Let $\quad \Gamma_{\wp}=\left\{\wp \mid V^{\zeta}(\wp) \leq \varrho /(1-\varnothing) \tau\right\}$ and $\widetilde{\Gamma}_{\wp}=\left\{\wp \mid V^{\zeta}(\wp)>\varrho /(1-\varnothing) \tau\right\}$. Discuss the following two cases:

(1) If the initial value satisfies $\wp(t) \in \widetilde{\Gamma}_{\wp}$, then one has

$$
\dot{V}(\wp) \leq-\varnothing \tau V^{\zeta}(\wp) .
$$

According to equation (19), it holds that

$$
\int_{t_{0}}^{T} \frac{V(\wp)}{V^{\zeta}} d t \leq \int_{t_{0}}^{T} \varnothing \tau d t
$$

Then, we can get the following inequality:

$$
\frac{1}{1-\zeta} V^{1-\zeta}(\wp(T))-\frac{1}{1-\zeta} V^{1-\zeta}\left(\wp\left(t_{0}\right)\right) \leq-\varnothing \tau T
$$

Let

$$
T_{r}=\frac{1}{(1-\zeta) \varnothing \tau}\left[V^{1-\zeta}\left(\wp\left(t_{0}\right)\right)-\left(\frac{\varrho}{(1-\varnothing) \tau}\right)^{1-\zeta / \zeta}\right]
$$

Then, for $\forall t \geq T$, combining equations (21) and (22) can be expressed as

$$
\wp(t) \in \Gamma_{\wp} .
$$

(2) If the initial value satisfies $\wp(t) \in \Gamma_{\wp}$, then according to the first case, the trajectory of $\wp(t)$ does not exceed $\Gamma_{\wp}$. Then, it can be obtained that there exists $T_{r}<\infty$, $\forall t \geq T$, such that $\wp(t) \in \Gamma_{\wp}$. That is, the solution of the nonlinear system $\dot{\wp}=f(\wp)$ is SGPFS.

\section{Main Results}

3.1. Adaptive RBFNN Controller Design. Before we begin designing, coordinate transformation is introduced as follows:

$$
z_{1}=x_{1}-q_{d}, z_{2}=x_{2}-\alpha_{1},
$$

where $\alpha_{1}$ is a virtual controller defined as

$$
\alpha_{i}=\frac{b_{1}}{N}\left(-\frac{1}{2 a_{i}^{2}} z_{i} \widehat{\theta}_{i} \hbar_{i}\left(Z_{i}\right)^{T} \hbar_{i}\left(Z_{i}\right)-\frac{1}{2} z_{i}-k_{i} z_{i}^{2 \zeta-1}\right),
$$

where $\zeta>0, k_{i}>0$, and $a_{i}>0$ are design parameters and the $i$ th RBFNN $\hbar_{i}\left(Z_{i}\right)$ can identify the unknown nonlinear function in the design process. Define the unknown constant $\theta_{i}=\left\|\Upsilon_{i}^{*}\right\|^{2} / b_{i}$, where $\hat{\theta}_{i}$ is the estimation of $\theta_{i}$ and the estimation error is $\widetilde{\theta}_{i}=\theta_{i}-\widehat{\theta}_{i}$.

Choose the control law $\mathcal{u}$, the auxiliary controller $\bar{u}$, and the adaptive law as follows:

$$
u=\hat{e} \bar{u},
$$

$$
\bar{u}=-\frac{1}{2 a_{i}^{2}} z_{i} \widehat{\theta}_{i} \hbar_{i}\left(Z_{i}\right)^{T} \hbar_{i}\left(Z_{i}\right)-\frac{1}{2} z_{i}-k_{i} z_{i}^{2 \zeta-1},
$$

$$
\dot{\hat{\theta}}_{i}=\frac{r_{i}}{2 a_{i}^{2}} z_{i}^{2} \hbar_{i}\left(Z_{i}\right)^{T} \hbar_{i}\left(Z_{i}\right)-\beta_{i} \widehat{\theta}_{i},
$$

where $r_{i}>0$ and $\sigma_{i}>0$. We assume that $\widehat{\theta}_{i}(t) \geq 0$ in this article, $\widehat{e}=1 / \widehat{\mu}_{1}$ is the estimation of $\widehat{e}=1 / \widehat{\mu}_{1}$, and $\widetilde{e}=e-\widehat{e}$ is the estimated error. Select $\dot{\hat{e}}$ as

$$
\dot{\hat{e}}=-\varrho z_{n} b_{n} \bar{u}-\varrho_{0} \widehat{e},
$$

where $\varrho$ and $\varrho_{0}$ are the design constants.

Step 1: Lyapunov function candidate will be chosen as follows:

$$
V_{1}=\frac{1}{2} z_{1}^{2}+\frac{b_{1}}{2 r_{1}} \tilde{\theta}_{1}^{2}
$$

Differentiating $V_{1}$ yields

$$
\dot{V}_{1}=z_{1}\left(N x_{2}+\omega(t)-C-q_{d}\right)-\frac{b_{1}}{r_{1}} \widetilde{\theta}_{1} \dot{\hat{\theta}}_{1} .
$$

Choose the function $\hat{f}_{1}\left(Z_{1}\right)$ defined by

$$
\hat{f}_{1}\left(Z_{1}\right)=-C+\omega(t)-\dot{y}_{d}+k_{1} z_{1}^{2 \zeta-1},
$$

then equation (29) can be rewritten as

$$
\dot{V}_{1}=z_{1}\left(\widehat{f}_{1}+N x_{2}\right)-\frac{b_{1}}{r_{1}} \tilde{\theta}_{1} \dot{\hat{\theta}}_{1}-k_{1} z_{1}^{2 \zeta}
$$

According to Lemma 1, RBFNN $\Upsilon_{1}^{* T} \hbar_{1}\left(Z_{1}\right)$ can be applied to identify the unknown function $\widehat{f}_{1}\left(Z_{1}\right)$. For any given $\varepsilon_{1}>0$,

$$
\hat{f}_{1}\left(Z_{1}\right)=\Upsilon_{1}^{* T} \hbar_{1}\left(Z_{1}\right)+\epsilon_{1}\left(Z_{1}\right),\left|\epsilon_{1}\left(Z_{1}\right)\right| \leq \varepsilon_{1},
$$

where $\epsilon_{1}\left(Z_{1}\right)$ is the approximation error. Consequently, let $\theta_{1}=\left\|\Upsilon_{1}^{*}\right\|^{2} / b_{1}$, and one can obtain 


$$
\begin{aligned}
z_{1} \widehat{f}_{1}\left(Z_{1}\right)= & z_{1} \frac{\Upsilon_{1}^{* T}}{\left\|\Upsilon_{1}^{*}\right\|}\left\|\Upsilon_{1}^{*}\right\| \hbar_{1}-z_{1} \delta_{1} \leq \frac{b_{1} z_{1}^{2}}{2 a_{1}^{2}} \frac{\left\|\Upsilon_{1}^{*}\right\|^{2}}{b_{1}} \hbar_{1}^{T} \hbar_{1} \\
& +\frac{1}{2} a_{1}^{2}+\frac{1}{2} b_{1} z_{1}^{2}+\frac{1}{2 b_{1}} \varepsilon_{1}^{2} \\
= & \frac{b_{1}}{2 a_{1}^{2}} z_{1}^{2} \theta_{1} \hbar_{1}^{T} \hbar_{1}+\frac{1}{2} a_{1}^{2}+\frac{1}{2} b_{1} z_{1}^{2}+\frac{1}{2 b_{1}} \varepsilon_{1}^{2} .
\end{aligned}
$$

Combining equations (31) and (33) gives

$$
\begin{aligned}
\dot{V}_{1} \leq & z_{1}\left(\frac{b_{1}}{2 a_{1}^{2}} z_{1} \theta_{1} \hbar_{1}^{T} \hbar_{1}+N \alpha_{1}\right)+N z_{1} z_{2}-\frac{b_{1}}{r_{1}} \tilde{\hat{\theta}}_{1} \\
& -k_{1} z_{1}^{2 \zeta}+\frac{1}{2} a_{1}^{2}+\frac{1}{2} b_{1} z_{1}^{2}+\frac{1}{2 b_{1}} \varepsilon_{1}^{2} .
\end{aligned}
$$

According to equation (24) and Assumption 4, it holds that

$$
z_{1} N \alpha_{1} \leq-\frac{b_{1}}{2 a_{1}^{2}} z_{1}^{2} \widehat{\theta}_{1} \hbar_{1}^{T} \hbar_{1}-k_{1} b_{1} z_{1}^{2 \zeta}-\frac{1}{2} b_{1} z_{1}^{2}
$$

Combining (36) with (37) gives

$$
\begin{aligned}
\dot{V}_{1} \leq & \frac{b_{1}}{2 a_{1}^{2}} z_{1}^{2} \theta_{1} \hbar_{1}^{T} \hbar_{1}-\frac{b_{1}}{2 a_{1}^{2}} z_{1}^{2} \widehat{\theta}_{1} \hbar_{1}^{T} \hbar_{1}-k_{1}\left(1+b_{1}\right) z_{1}^{2 \zeta} \\
& +N z_{1} z_{2}-\frac{b_{1}}{r_{1}} \tilde{\theta}_{\hat{\theta}}+\frac{1}{2} a_{1}^{2}+\frac{1}{2 b_{1}} \varepsilon_{1}^{2} .
\end{aligned}
$$

Choose the adaptation law as

$$
\dot{\hat{\theta}}_{1}=\frac{r_{1}}{2 a_{1}^{2}} z_{1}^{2} \hbar_{1}\left(Z_{1}\right)^{T} \hbar_{1}\left(Z_{1}\right)-\beta_{i} \widehat{\theta}_{1}
$$

According to equation (37), it holds that

$$
\frac{b_{1}}{r_{1}} \tilde{\theta} \dot{\hat{\theta}}_{1}=\frac{b_{1}}{2 a_{1}^{2}} z_{1}^{2} \tilde{\theta}_{1} \hbar_{1}^{T} \hbar_{1}-\frac{b_{1} \beta_{1}}{r_{1}} \widetilde{\theta}_{1} \widehat{\theta}_{1}
$$

It is noted that

$$
\frac{b_{1} \beta_{1}}{r_{1}} \widetilde{\theta}_{1} \widehat{\theta}_{1} \leq-\frac{b_{1} \beta_{1}}{2 r_{1}} \widetilde{\theta}_{1}^{2}+\frac{b_{1} \beta_{1}}{2 r_{1}} \theta_{1}^{2} .
$$

Therefore, combining equations (38), (40), and (41) can be expressed as

$$
\dot{V}_{1} \leq \ell_{1}+N z_{1} z_{2}
$$

where

$$
\ell_{1}=-k_{1}\left(1+b_{1}\right) z_{1}^{2 \zeta}+\frac{b_{1} \beta_{1}}{2 r_{1}}\left(-\widetilde{\theta}_{1}^{2}+\theta_{1}^{2}\right)+\frac{1}{2} a_{1}^{2}+\frac{1}{2 b_{1}} \varepsilon_{1}^{2} .
$$

Step 2: choose a Lyapunov function candidate as

$$
V_{2}=V_{1}+\frac{1}{2} z_{2}^{2}+\frac{b_{2}}{2 r_{2}} \widetilde{\theta}_{2}^{2}+\frac{\mu_{1}}{2 \varrho} \widetilde{e}^{2}
$$

It is that

$$
\begin{aligned}
\dot{V}_{2}= & \ell_{1}+N z_{1} z_{2}-\frac{b_{2}}{r_{2}} \widetilde{\theta}_{2} \dot{\hat{\theta}}_{2} \\
& -\frac{\mu_{1}}{\varrho} \tilde{e} \dot{\hat{e}}+z_{2}\left(f(x)+g(x) H(\bar{u})+\psi(x) \omega(t)-\dot{\alpha}_{1}\right) .
\end{aligned}
$$

Establish the actual controller as

$$
\bar{u}=-\frac{1}{2 a_{2}^{2}} z_{2} \widehat{\theta}_{2} \hbar_{2}\left(Z_{2}\right)^{T} \hbar_{2}\left(Z_{2}\right)-\frac{1}{2} z_{2}-k_{2} z_{2}^{2 \zeta-1} .
$$

According to equations and Assumption 4, one has

$$
\begin{gathered}
z_{2} g(x) H(\bar{u})-\frac{\mu}{\varrho} \tilde{e} \dot{\hat{e}} \leq z_{2} b_{2} \bar{u}-z_{2} b_{2} \mu_{1} \widetilde{e} \bar{u}+z_{2} b_{2} \mu_{2} \iota \\
-\frac{\mu_{1}}{\varrho} \widetilde{e}\left(-\varrho z_{2} b_{2} \bar{u}-\varrho_{0} \widehat{e}\right) \\
\leq z_{2} b_{2} \bar{u}+z_{2} b_{2} \mu_{2} \iota+\frac{\mu_{1}}{\varrho} \widetilde{e} \widehat{e} \leq-\frac{1}{2 a_{2}^{2}} z_{2}^{2} b_{2} \widehat{\theta}_{2} \hbar_{2}^{T} \hbar_{2} \\
-\frac{1}{2} b_{2} z_{2}^{2}-k_{2} b_{2} z_{2}^{2 \zeta}+\frac{1}{2}\left(b_{2} z_{2}\right)^{2}+\frac{1}{2}\left(\mu_{2} \bar{l}\right)^{2}+\frac{\varrho_{0}}{2 \varrho \mu_{1}},
\end{gathered}
$$

where $\bar{\imath}=\sqrt{[n]} 1 /(\vartheta+\xi)$.

Now, substituting inequalities (47) into (45) results in

$$
\begin{aligned}
\dot{V}_{2} \leq & \ell_{1}+z_{2} \widehat{f}_{2}-\frac{1}{2 a_{2}^{2}} z_{2}^{2} b_{2} \widehat{\theta}_{2} \hbar_{2}^{T} \hbar_{2}-\frac{1}{2} b_{2} z_{2}^{2}-k_{2} b_{2} z_{2}^{2 \zeta} \\
& -k_{2} z_{2}^{2 \zeta}+\frac{1}{2}\left(\mu_{2} \bar{l}\right)^{2}+\frac{\varrho_{0}}{2 \varrho \mu_{1}}-\frac{b_{2}}{r_{2}} \widetilde{\theta}_{2} \dot{\hat{\theta}}_{2},
\end{aligned}
$$

where $\hat{f}_{2}=f(x)+k_{2} z_{2}^{2 \zeta}+N z_{1}+\psi(x) \omega(t)+(1 / 2) b_{2}^{2} z_{2}^{2}-$ $\dot{\alpha}_{1}$, which can be estimated by $\operatorname{RBFNN} \Upsilon_{2}^{* T} \hbar_{2}\left(Z_{2}\right)$ for any given positive constant $\varepsilon_{2}$. Similarly, let $\theta_{2}=\left\|\Upsilon_{2}^{*}\right\|^{2} / b_{2}$, and one easily obtains 


$$
\begin{aligned}
z_{2} \hat{f}_{2}\left(Z_{2}\right)= & z_{2} \frac{\Upsilon_{2}^{* T}}{\left\|\Upsilon_{2}^{*}\right\|}\left\|\Upsilon_{2}^{*}\right\| \hbar_{2}-z_{1} \delta_{2} \leq \frac{b_{2} z_{2}^{2}}{2 a_{2}^{2}} \frac{\left\|\Upsilon_{2}^{*}\right\|^{2}}{b_{1}} \hbar_{2}^{T} \hbar_{2} \\
& +\frac{1}{2} a_{2}^{2}+\frac{1}{2} b_{2} z_{2}^{2}+\frac{1}{2 b_{2}} \varepsilon_{2}^{2} \\
= & \frac{b_{2}}{2 a_{2}^{2}} z_{2}^{2} \theta_{2} \hbar_{2}^{T} \hbar_{2}+\frac{1}{2} a_{2}^{2}+\frac{1}{2} b_{1} z_{2}^{2}+\frac{1}{2 b_{2}} \varepsilon_{2}^{2} .
\end{aligned}
$$

Then, equation (46) can be rewritten as

$$
\begin{aligned}
\dot{V}_{2} \leq & \ell_{1} \\
+ & \frac{b_{2}}{2 a_{2}^{2}} z_{2}^{2} \theta_{2} \hbar_{2}^{T} \hbar_{2}-\frac{1}{2 a_{2}^{2}} z_{2}^{2} b_{2} \widehat{\theta}_{2} \hbar_{2}^{T} \hbar_{2} \\
& -k_{2}\left(1+b_{2}\right) z_{2}^{2 \zeta}+\frac{1}{2} a_{2}^{2}+\frac{1}{2 b_{2}} \varepsilon_{2}^{2}+\frac{1}{2}\left(\mu_{2} \bar{\imath}\right)^{2}+\frac{\varrho_{0}}{2 \varrho \mu_{1}}-\frac{b_{2}}{r_{2}} \widetilde{\theta}_{2} \dot{\hat{\theta}}_{2} .
\end{aligned}
$$

\subsection{Stability Analysis}

Theorem 1. Consider system (4). Suppose Assumptions 2-4 hold, then all the signals are SGPFS, under any bounded initial conditions when controller (25) and adaptive law (28) are employed.

Proof. Choose the adaptation law as

$$
\dot{\hat{\theta}}_{2}=\frac{r_{2}}{2 a_{2}^{2}} z_{2}^{2} \hbar_{1}\left(Z_{2}\right)^{T} \hbar_{1}\left(Z_{2}\right)-\beta_{i} \widehat{\theta}_{2} .
$$

According to equation (49), it holds that

$$
\frac{b_{2}}{r_{2}} \tilde{\theta} \dot{\hat{\theta}}_{2}=\frac{b_{2}}{2 a_{2}^{2}} z_{2}^{2} \widetilde{\theta}_{2} \hbar_{2}^{T} \hbar_{2}-\frac{b_{2} \beta_{2}}{r_{2}} \widetilde{\theta}_{2} \widehat{\theta}_{2} .
$$

It is noted that

$$
\frac{b_{2} \beta_{2}}{r_{2}} \widetilde{\theta}_{2} \widehat{\theta}_{2} \leq-\frac{b_{2} \beta_{2}}{2 r_{2}} \widetilde{\theta}_{2}^{2}+\frac{b_{2} \beta_{2}}{2 r_{2}} \theta_{2}^{2}
$$

Substituting (52) and (53) into (50), it gives

$$
\begin{aligned}
\dot{V}_{2} \leq \ell_{1} & +\frac{b_{2} \beta_{2}}{r_{2}} \widetilde{\theta}_{2} \widehat{\theta}_{2}-k_{2} b_{2} z_{2}^{2 \zeta}-k_{2} z_{2}^{2 \zeta}+\frac{1}{2}\left(\mu_{2} \bar{\iota}\right)^{2} \\
& +\frac{\varrho_{0}}{2 \varrho \mu_{1}}+\frac{1}{2} a_{2}^{2}+\frac{1}{2 b_{2}} \varepsilon_{2}^{2} .
\end{aligned}
$$

Then, we can get

$$
\begin{aligned}
\dot{V}_{2} \leq & -\sum_{i=1}^{2} k_{i}\left(1+b_{i}\right) z_{i}^{2 \zeta}+\frac{b_{1} \beta_{1}}{2 r_{1}}\left(-\widetilde{\theta}_{1}^{2}+\theta_{1}^{2}\right) \\
& +\frac{b_{2} \beta_{2}}{2 r_{2}}\left(-\widetilde{\theta}_{2}^{2}+\theta_{2}^{2}\right)+\frac{1}{2}\left(a_{1}^{2}+\frac{1}{b_{1}} \varepsilon_{1}^{2}\right) \\
& +\frac{1}{2}\left(a_{2}^{2}+\frac{1}{b_{2}} \varepsilon_{2}^{2}\right)+\frac{1}{2}\left(\mu_{2} \bar{\imath}\right)^{2}+\frac{\varrho_{0}}{2 \varrho \mu_{1}} .
\end{aligned}
$$

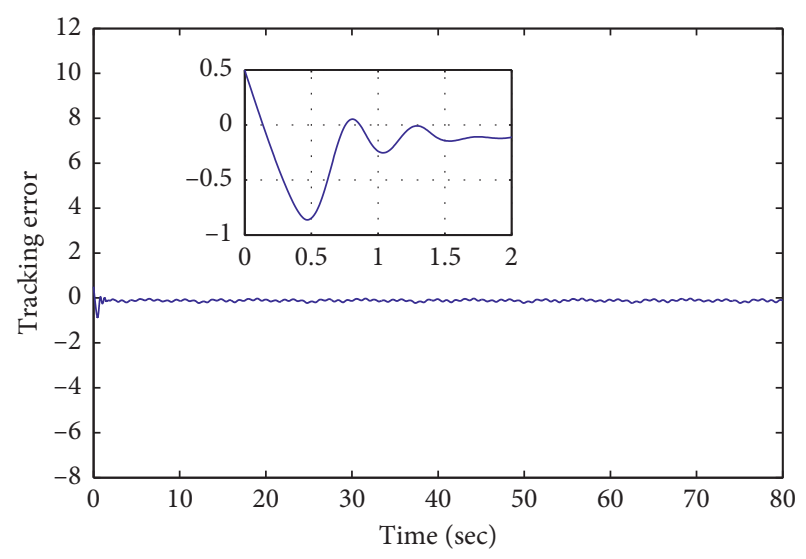

FIgURE 1: Tracking error $q(t)-q_{d}$.

Let $k_{\text {min }}=\min _{1 \leq i \leq 2}\left\{k_{i}\left(1+b_{i}\right)\right\}$, and by Lemma 3 , it is deduced that

$$
-\sum_{i=1}^{2} k_{i}\left(1+b_{i}\right) z_{i}^{2 \zeta} \leq-k_{\min } \sum_{i=1}^{2} z_{i}^{2 \zeta} \leq-\bar{K}\left(\frac{1}{2} \sum_{i=1}^{2} z_{i}^{2}\right)^{\zeta},
$$

where $\bar{K}=2^{\zeta} k_{\min }$.

Let $\beta_{\min }=\min _{1 \leq i \leq 2}\left\{\beta_{i}\right\}$, and by taking equation (54) into account, inequality (55) can be rewritten as

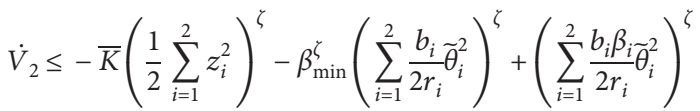

$$
\begin{aligned}
& +\frac{b_{1} \beta_{1}}{2 r_{1}}\left(-\tilde{\theta}_{1}^{2}+\theta_{1}^{2}\right)+\frac{b_{2} \beta_{2}}{2 r_{2}}\left(-\tilde{\theta}_{2}^{2}+\theta_{2}^{2}\right) \\
& +\frac{1}{2}\left(a_{1}^{2}+\frac{1}{b_{1}} \varepsilon_{1}^{2}\right)+\frac{1}{2}\left(a_{2}^{2}+\frac{1}{b_{2}} \varepsilon_{2}^{2}\right)+\frac{1}{2}\left(\mu_{2} \bar{l}\right)^{2}+\frac{\varrho_{0}}{2 \varrho \mu_{1}} \leq-\lambda V^{\zeta} \\
& +\left(\sum_{i=1}^{2} \frac{b_{i} \beta_{i} \widetilde{\theta}_{i}^{2}}{2 r_{i}}\right)^{\zeta}+\frac{b_{1} \beta_{1}}{2 r_{1}}\left(-\widetilde{\theta}_{1}^{2}+\theta_{1}^{2}\right)+\frac{b_{2} \beta_{2}}{2 r_{2}}\left(-\widetilde{\theta}_{1}^{2}+\theta_{1}^{2}\right) \\
& +\frac{1}{2}\left(a_{1}^{2}+\frac{1}{b_{1}} \varepsilon_{1}^{2}\right) \\
& +\frac{1}{2}\left(a_{2}^{2}+\frac{1}{b_{2}} \varepsilon_{2}^{2}\right)+\frac{1}{2}\left(\mu_{2} \bar{l}\right)^{2}+\frac{\varrho_{0}}{2 \varrho \mu_{1}},
\end{aligned}
$$

where $\lambda=\min \left\{\bar{K}, \beta_{\min }^{\zeta}\right\}$.

By Lemma 2, let $\mu=\zeta, \rho=1-\zeta, \lambda=\zeta^{-1}, \phi=$ $\sum_{i=1}^{2} b_{i} \beta_{i} / 2 r_{i} \widetilde{\theta}_{i}^{2}$, and $\psi=1$, then we get

$$
\left(\sum_{i=1}^{2} \frac{b_{i} \beta_{i}}{2 r_{i}} \tilde{\theta}_{i}^{2}\right)^{\zeta} \leq \sum_{i=1}^{2} \frac{b_{i} \beta_{i}}{2 r_{i}} \tilde{\theta}_{i}^{2}+(1-\zeta) \zeta^{\zeta / 1-\zeta} .
$$

Then, equation (55) becomes

$$
\dot{V}_{2} \leq-\lambda V^{\zeta}+\varrho,
$$

where $\varrho=(1-\zeta) \zeta^{\zeta / 1-\zeta}+\sum_{i=1}^{2} \quad b_{i} \beta_{i} / 2 r_{i} \theta_{i}^{2}+(1 / 2)\left(a_{1}^{2}+\right.$ $\left.\left(1 / b_{1}\right) \varepsilon_{1}^{2}\right)+(1 / 2)\left(a_{2}^{2}+\left(1 / b_{2}\right) \varepsilon_{2}^{2}\right)+(1 / 2)\left(\mu_{2} \bar{l}\right)^{2}+\varrho_{0} / 2 \varrho \mu_{1}$. 
Define a positive constant $\partial$ as follows:

$$
\partial=\frac{\varrho}{\left(1-\theta_{0}\right) \lambda} .
$$

Equation (57) can be expressed as

$$
V^{\zeta}(z(t), \bar{\theta}(t)) \leq \frac{\varrho}{\left(1-\theta_{0}\right) \lambda} .
$$

For $t \geq T_{\text {reach }}$, let

$$
T_{\text {reach }}=\frac{1}{\left(1-\theta_{0}\right) \lambda}\left[V^{1-\zeta}(z(0), \bar{\theta}(0))-\left(\frac{\varrho}{\left(1-\theta_{0}\right) \lambda}\right)^{1-\zeta / \zeta}\right] \text {, }
$$

with $z(0)=\left[z_{1}(0), z_{2}(0)\right]^{T}$ and $\bar{\theta}(0)=\left[\theta_{1}(0), \theta_{2}(0)\right]^{T}$.

Therefore, all the signals are SGPFS.

Then, for $t \geq T_{\text {reach }}$, we can obtain that

$$
\left|y-q_{d}\right| \leq 2\left(\frac{\varrho}{\left(1-\theta_{0}\right) \lambda}\right)^{1 / 2 \zeta} .
$$

Then, the proof is completed.

\section{Simulation Example}

In this section, to certify the feasibility of the strategy presented in this work, a simulation example is given by MATLAB.

Select the system parameters and external disturbance in system (4) as

$$
\begin{aligned}
& N=1, R=6 \mathrm{~s}, C=2.5 \text { packets } / \mathrm{s}, \\
& a_{1}=0.3, a_{2}=2, k_{1}=k_{2}=0.1, \\
& r_{1}=r_{2}=20, \beta_{1}=0.08, \beta_{2}=0.2, \\
& \omega=0.5 e^{-0.5 t} \sin (6 t) .
\end{aligned}
$$

Define the hysteresis $H(u)$ as follows:

$$
H(u)=\mu_{1} u+\mu_{2} l,
$$

where $\mu_{1}=10$ and $\mu_{2}=0.5 . \iota$ is selected as equation (8). The function $f(\iota, \dot{u})$ is selected as equation (7).

The initial values of the state are $\vartheta=2, \xi=1$, and $n=3$; $y(0)=0.5$.

The results of simulation are shown in Figures 1-6. Figure 1 shows the tracking error of $q(t)$ and $q_{d}$. It is clear that the queue can track the desired queue within the allowable error. Figure 2 introduces the hysteresis output $H(u)$. Besides, Figures 3 and 4 show the trajectory of the adaptive law, from which we can obtain that all the adaptive laws are SGPFS. Figure 5 shows the trajectory of the rate $r(t)$. As a result, the proposed strategy is effective.

In addition, in order to illustrate the advantages of this algorithm, the simulation comparisons are made between the method in this paper and the random early detection (RED) algorithm. The comparison result is shown in Figure 6 , in which the preset properties with respect to $q(t)-q_{d}$ are obtained. Moreover, it should be pointed out that the maximum overshoot of $q(t)-q_{d}$ is less than 0.8 . Further, it

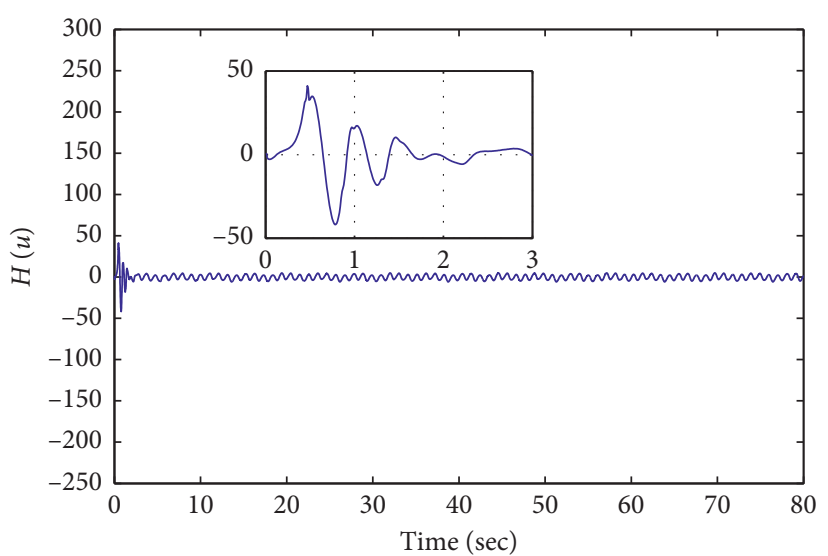

Figure 2: The hysteresis output $H(u)$.

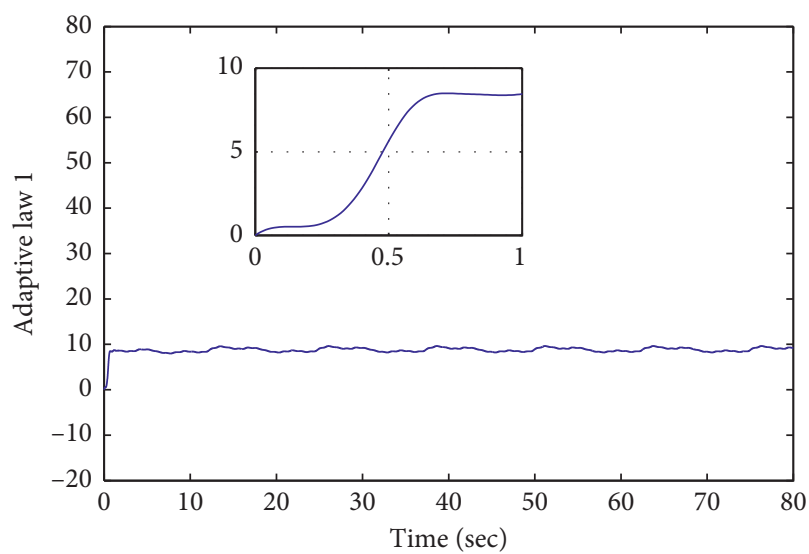

Figure 3: The trajectory of the adaptive law 1.

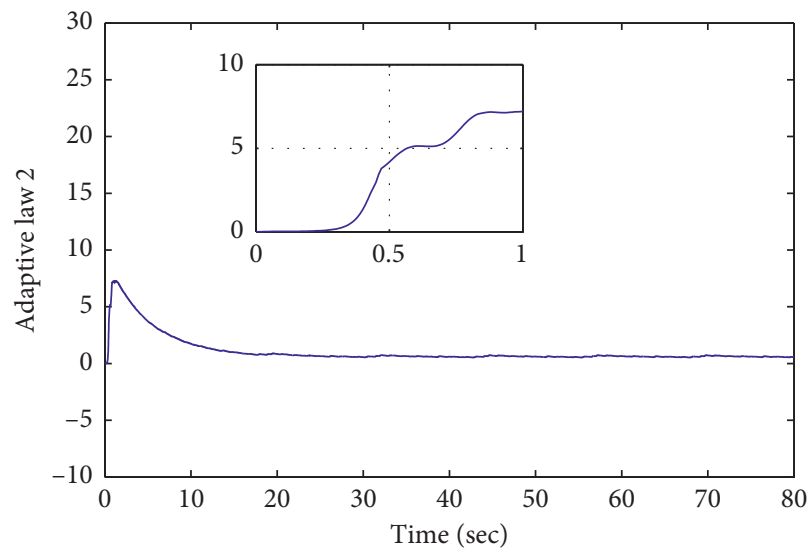

Figure 4: The trajectory of the adaptive law 2.

is easy to observe from Figure 6 that the smaller overshoot and the less chattering are achieved comparing with RED algorithm. As a result, the proposed method has the better performances. 


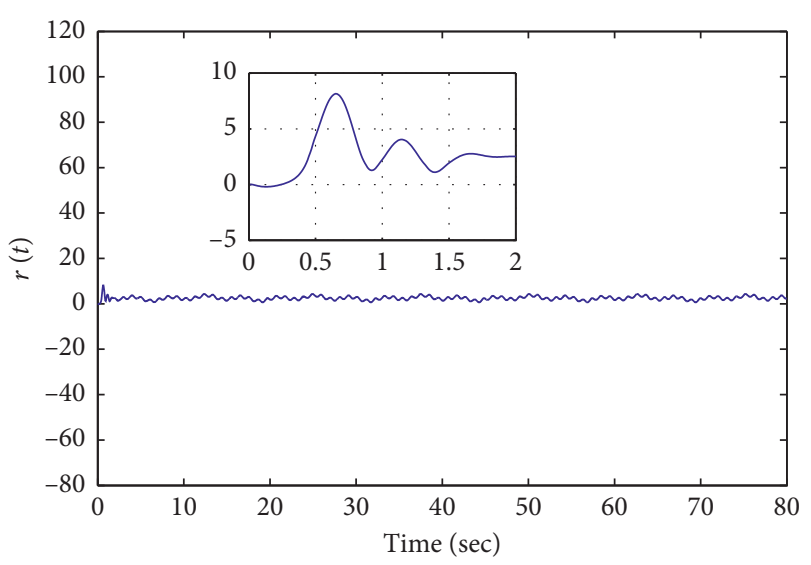

FIgURE 5: The sending rate $r(t)$.

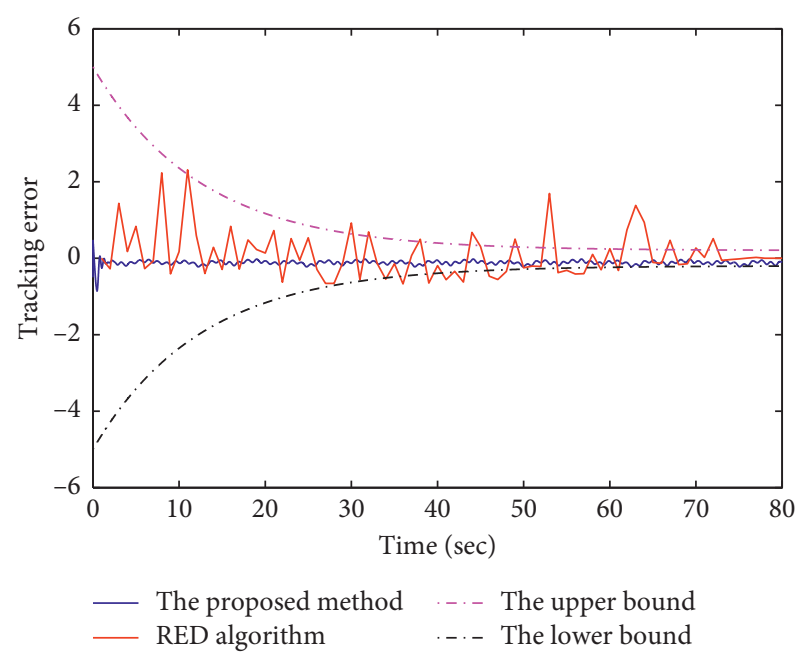

FIgURE 6: The comparison results with the tracking error.

\section{Conclusion}

In this manuscript, an adaptive FT control is considered for TCP/AQM networks. The finite-time controller by using the backstepping technique and the adaptive neural control method ensures that the queue length tracks the desired queue length and the tracking error converges to the prescribed area. Besides, the controller can not only reduce the influence of the disturbance, but also shorten the impression of uncertainty. Finally, an example is offered to verify the superiority and effectiveness.

\section{Data Availability}

The raw data supporting the conclusions of this article will be made available by the authors, without undue reservation, to any qualified researcher.

\section{Conflicts of Interest}

The authors declare that there are no conflicts of interest regarding the publication of this paper.

\section{Acknowledgments}

This research work was supported financially by the National Natural Science Foundation of China (Grant no. 11974373).

\section{References}

[1] K. Wang, Y. Liu, X. Liu, Y. Jing, and G. M. Dimirovski, "Study on TCP/AQM network congestion with adaptive neural network and barrier Lyapunov function," Neurocomputing, vol. 363, pp. 27-34, 2019.

[2] V. Jacobson, "Congestion avoidance and control," $A C M$ SIGCOMM Computer Communication Review, vol. 18, no. 4, pp. 314-329, 1988.

[3] B.-M. Laws and L. Larry, "TCP Vegas: end-to-end congestion avoidance on a global Internet," IEEE Journal on Selected Areas in Communications, vol. 13, pp. 1465-1480, 1995.

[4] M. Mathis, J. Mahdavi, S. Floyd et al., "TCP selective acknowledgment options," RFC, vol. 2018, 1996.

[5] S. Floyd and T. Henderson, "The new reno modification to TCPs fast recovery algorithm," RFC, vol. 2582, 1999.

[6] B. Braden and D. Clark, "Recommendations on queue management and congestion avoidance in the Internet," RFC, vol. 2309, 1998.

[7] S. Floyd and V. Jacobson, "Random early detection gateways for congestion avoidance," IEEE/ACM Transactions on Networking, vol. 1, no. 4, pp. 397-413, 1993.

[8] G. Feng, A. K. Agarwal, A. Jayaraman, and C. K. Siew, "Modified RED gateways under bursty traffic," IEEE Communications Letters, vol. 8, no. 5, pp. 323-325, 2004.

[9] F. Anjum and L. Tassiulas, "Fair bandwidth sharing among adaptive and non-adaptive flows in the internet," IEEE, vol. 8, pp. 1412-1420, 1999.

[10] V. Misra, W. B. Gong, and D. Towsley, "Fluid-based analysis of a network of AQM routers supporting TCP flows with an application to RED," IEEE, vol. 51, pp. 151-160, 2000.

[11] L. Tan, W. Zhang, G. Peng, and G. Chen, "Stability of TCP/ RED systems in AQM routers," IEEE Transactions on Automatic Control, vol. 51, pp. 393-1398, 2006.

[12] J. Sun, K. Ko, G. Chen, S. Chan, and M. Zukerman, "PD-RED: to improve the performance of RED," IEEE Communications Letters, vol. 7, pp. 406-408, 2003.

[13] C. Hollot, V. Misra, D. Towsley, and W. Gong, On Designing Improved Controllers for AQM Routers Supporting TCP Flows, pp. 1726-1734, Springer, Anchorage, AK, USA, 2001.

[14] T. Wei and S. Zhang, "AQM algorithm based on nonlinear control method," Journal of Communication, vol. 30, pp. 58-67, 2009.

[15] C.-K. Chen, Y.-C. Hung, T.-L. Liao, and J.-J. Yan, "Design of robust active queue management controllers for a class of TCP communication networks," Information Sciences, vol. 177, no. 19, pp. 4059-4071, 2007.

[16] L.-S. Tan, C. Yuan, and M. Zukerman, "A price-based Internet congestion control scheme," IEEE Communications Letters, vol. 12, pp. 331-333, 2008.

[17] W. Zhang, L. Tan, and G. Peng, "Dynamic queue level control of TCP/RED systems in AQM routers," Computers \& Electrical Engineering, vol. 35, no. 1, pp. 59-70, 2009.

[18] W. Zhang, L. Tan, C. Yuan, G. Chen, and F. Ge, "Internet primal-dual congestion control: stability and applications," Control Engineering Practice, vol. 21, no. 1, pp. 87-95, 2013.

[19] Y. Liu, X. Liu, Y. Jing, and S. Zhou, “Adaptive backstepping Ho tracking control with prescribed performance for internet congestion," ISA Transactions, vol. 72, pp. 92-99, 2018. 
[20] D. Ghosh, K. Jagannathan, and G. Raina, "Right buffer sizing matters: some dynamical and statistical studies on Compound TCP,” Performance Evaluation, vol. 139, p. 102095, 2020.

[21] R. K. Chaturvedi and S. Chand, "Optimal load balancing linked increased algorithm for multipath TCP," Wireless Personal Communications, vol. 111, no. 3, pp. 1505-1524, 2019.

[22] J. Zhao, J.-C. Liu, H.-Y. Wang, and C. Xu, "Measurement, analysis, and enhancement of multipath TCP energy efficiency for datacenters," IEEE Transactions on Automatic Control, vol. 28, pp. 57-70, 2019.

[23] G. Tao and P. V. Kokotovic, "Adaptive control of plants with unknown hystereses," IEEE Transactions on Automatic Control, vol. 40, no. 2, pp. 200-212, 1995.

[24] J.-S. Pan, C.-Y. Lee, A. Sghaier, M. Zeghid, and J. Xie, "Novel systolization of subquadratic space complexity multipliers based on toeplitz matrix-vector product approach," IEEE Transactions on Very Large Scale Integration (VLSI) Systems, vol. 27, no. 7, pp. 1614-1622, 2019.

[25] J. Zhou, C. Wen, and T. Li, "Adaptive output feedback control of uncertain nonlinear systems with hysteresis nonlinearity," IEEE Transactions on Automatic Control, vol. 57, no. 10, pp. 2627-2633, 2012.

[26] C.-Y. Su, Y. Stepanenko, J. Svoboda, and T. P. Leung, "Robust adaptive control of a class of nonlinear systems with unknown backlash-like hysteresis," IEEE Transactions on Automatic Control, vol. 45, no. 12, pp. 2427-2432, 2000.

[27] X. Liu, Y. Li, and Y. Li, "Adaptive tracking control for a class of uncertain switched stochastic nonlinear systems," IEEE Transactions on Automatic Control, vol. 2019, p. 33, 2019.

[28] F. Wang, Z. Liu, Y. Zhang, and C. L. P. Chen, "Adaptive fuzzy control for a class of stochastic pure-feedback nonlinear systems with unknown hysteresis," IEEE Transactions on Fuzzy Systems, vol. 24, no. 1, pp. 140-152, 2016.

[29] P. Dorato, An Overview of Finite-Time Stability, pp. 185-194, Springer, Boston, MA, USA, 2006.

[30] S. P. Bhat and D. S. Bernstein, "Finite-time stability of continuous autonomous systems," SIAM Journal on Control and Optimization, vol. 38, no. 3, pp. 751-766, 2000.

[31] W. Lv, F. Wang, and Y. Li, "Finite-time adaptive fuzzy outputfeedback control of MIMO nonlinear systems with hysteresis," Neurocomputing, vol. 296, pp. 74-81, 2018.

[32] S. P. Bhat and D. S. Bernstein, "Continuous finite-time stabilization of the translational and rotational double integrators," IEEE Transactions on Automatic Control, vol. 43, no. 5, pp. 678-682, 1998.

[33] L. Zou, Z. Wang, H. Gao, and F. E. Alsaadi, "Finite-horizon consensus control of time-varying multiagent systems with stochastic communication protocol," IEEE Transactions on Cybernetics, vol. 47, no. 8, pp. 1830-1840, 2017.

[34] F. Wang, B. Chen, X. Liu, and C. Lin, "Finite-time adaptive fuzzy tracking control design for nonlinear systems," IEEE Transactions on Fuzzy Systems, vol. 26, no. 3, pp. 1207-1216, 2018.

[35] A. Elham and S. M. Vahid, Robust Congestion Control for TCP/AQM Using Integral Backstepping Control, pp. 18401844, IEEE, New York, NY, USA, 2015.

[36] V. Nekoukar and A. Erfanian, "Adaptive fuzzy terminal sliding mode control for a class of MIMO uncertain nonlinear systems," Fuzzy Sets and Systems, vol. 179, no. 1, pp. 34-49, 2011.

[37] H. Liu and T. Zhang, "Neural network-based robust finitetime control for robotic manipulators considering actuator dynamics," Robotics and Computer-Integrated Manufacturing, vol. 29, no. 2, pp. 301-308, 2013.

[38] H. Liu and T. Zhang, "Adaptive neural network finite-time control for uncertain robotic manipulators," Journal of Intelligent \& Robotic Systems, vol. 75, no. 3-4, pp. 363-377, 2014.

[39] Y. Cui, M. Fei, and D. Du, "Design of a robust observer-based memorylessHoocontrol for internet congestion," International Journal of Robust and Nonlinear Control, vol. 26, no. 8, pp. 1732-1747, 2016.

[40] L.-X. Wang and J. M. Mendel, "Fuzzy basis functions, universal approximation, and orthogonal least-squares learning," IEEE Transactions on Neural Networks, vol. 3, no. 5, pp. 807-814, 1992.

[41] C. Qian and W. Lin, "Non-Lipschitz continuous stabilizers for nonlinear systems with uncontrollable unstable linearization," Systems \& Control Letters, vol. 42, no. 3, pp. 185-200, 2001.

[42] Z. Zhu, Y. Xia, and M. Fu, "Attitude stabilization of rigid spacecraft with finite-time convergence," International Journal of Robust and Nonlinear Control, vol. 21, no. 6, pp. 686-702, 2011.

[43] S. Yu, X. Yu, B. Shirinzadeh, and Z. Man, "Continuous finitetime control for robotic manipulators with terminal sliding mode," Automatica, vol. 41, no. 11, pp. 1957-1964, 2005.

[44] W.-S. Lv and F. Wang, "Finite-time adaptive fuzzy tracking control for a class of nolinear systems with unknown hysteresis," International Journal of Robust and Nonlinear Control, vol. 2, pp. 1-9, 2017. 